\title{
POC QUESTIONNAIRE
}

ASSESSMENT OF THE IMPACT OF ENHANCED WORKFLOW PATTERNS ASSOCIATED WITH UPFRONT, EARLY POINT-OF-CARE TESTING ON WAITING AND DISPOSITION TIMES IN AN EMERGENCY DEPARTMENT

\section{Please circle the option you feel is the most correct}

\section{Regarding patients seen in YELLOW MEDICAL AREA}

\begin{tabular}{|l|c|c|c|c|c|}
\hline & $\begin{array}{c}\text { Strongly } \\
\text { Disagree }\end{array}$ & Disagree & Neutral & Agree & $\begin{array}{c}\text { Strongly } \\
\text { Agree }\end{array}$ \\
\hline $\begin{array}{l}\text { It saved time when patients only had the } \\
\text { iSTAT (troponin, INR, Chem8, CG4+) } \\
\text { results when seen in Yellow Medical }\end{array}$ & 1 & 2 & 3 & 4 & 5 \\
\hline $\begin{array}{l}\text { It saved time when patients only had the } \\
\text { CBC (complete blood count) results } \\
\text { when seen in Yellow Medical }\end{array}$ & 1 & 2 & 3 & 4 & 5 \\
\hline $\begin{array}{l}\text { It saved time when patients only had the } \\
\text { LODOX when seen in Yellow Medical }\end{array}$ & 1 & 2 & 3 & 4 & 5 \\
\hline $\begin{array}{l}\text { It saved time when patients only had the } \\
\text { ECG when seen in Yellow Medical }\end{array}$ & 1 & 2 & 3 & 4 & 5 \\
\hline $\begin{array}{l}\text { It saved time when patients had all EPOC } \\
\text { tests when seen in Yellow Medical }\end{array}$ & 1 & 2 & 3 & 4 & 5 \\
\hline
\end{tabular}

\section{Regarding patients seen in ORANGE MEDICAL AREA}

\begin{tabular}{|l|c|c|c|c|c|}
\hline & $\begin{array}{c}\text { Strongly } \\
\text { Disagree }\end{array}$ & Disagree & Neutral & Agree & $\begin{array}{c}\text { Strongly } \\
\text { Agree }\end{array}$ \\
\hline $\begin{array}{l}\text { It saved time when patients only had the } \\
\text { iSTAT (troponin, INR, Chem8, CG4+) } \\
\text { results when seen in Orange Medical }\end{array}$ & 1 & 2 & 3 & 4 & 5 \\
\hline $\begin{array}{l}\text { It saved time when patients only had the } \\
\text { CBC (complete blood count) results } \\
\text { when seen in Orange Medical }\end{array}$ & 1 & 2 & 3 & 4 & 5 \\
\hline $\begin{array}{l}\text { It saved time when patients only had the } \\
\text { LODOX when seen in Orange Medical }\end{array}$ & 1 & 2 & 3 & 4 & 5 \\
\hline
\end{tabular}




\begin{tabular}{|c|c|c|c|c|c|}
\hline $\begin{array}{l}\text { It saved time when patients only had the } \\
\text { ECG when seen in Orange Medical }\end{array}$ & 1 & 2 & 3 & 4 & 5 \\
\hline $\begin{array}{l}\text { It saved time when patients had all EPOC } \\
\text { tests when seen in Orange Medical }\end{array}$ & 1 & 2 & 3 & 4 & 5 \\
\hline
\end{tabular}

\section{General}

\begin{tabular}{|l|c|c|c|c|c|}
\hline & $\begin{array}{c}\text { Strongly } \\
\text { Disagree }\end{array}$ & Disagree & Neutral & Agree & $\begin{array}{c}\text { Strongly } \\
\text { Agree }\end{array}$ \\
\hline $\begin{array}{l}\text { I found it helpful to assess patients who } \\
\text { already had test results available }\end{array}$ & 1 & 2 & 3 & 4 & 5 \\
\hline $\begin{array}{l}\text { I think certain tests like those used in } \\
\text { EPOC should be performed prior to the } \\
\text { patient being assessed by a doctor/nurse }\end{array}$ & 1 & 2 & 3 & 4 & 5 \\
\hline $\begin{array}{l}\text { The trial protocols provided a better } \\
\text { workflow in the ED }\end{array}$ & 1 & 2 & 3 & 4 & 5 \\
\hline $\begin{array}{l}\text { If available, I would want the trial } \\
\text { protocol to be permanently available in the } \\
\text { ED }\end{array}$ & 1 & 2 & 3 & 4 & 5 \\
\hline $\begin{array}{l}\text { Higher cost of early point-of-care testing } \\
\text { is worth the time saved }\end{array}$ & 1 & 2 & 3 & 4 & 5 \\
\hline
\end{tabular}

4. Please RANK the tests from MOST helpful (1) to LEAST helpful (4)

\begin{tabular}{|c|c|c|c|}
\hline iSTAT & CBC & LODOX & ECG \\
\hline & & & \\
\hline
\end{tabular}

5. Please RANK the COMBINATION of tests from MOST helpful (1) to LEAST helpful (7)

\begin{tabular}{|c|c|c|c|c|c|c|}
\hline iSTAT & iSTAT & iSTAT & iSTAT & iSTAT & ECG & iSTAT \\
\hline$+\mathrm{CBC}$ & $+\mathbf{E C G}$ & $\begin{array}{l}+\mathrm{CBC} \\
+\mathrm{ECG}\end{array}$ & + LODOX & $\begin{array}{c}+ \text { CBC } \\
+ \text { LODOX }\end{array}$ & + LODOX & $\begin{aligned} & + \text { ECG } \\
+ & \text { LODOX }\end{aligned}$ \\
\hline
\end{tabular}




\section{RESULTS PREDICTION}

\begin{tabular}{|l|c|c|c|c|c|}
\hline & $\begin{array}{c}\text { Strongly } \\
\text { Disagree }\end{array}$ & Disagree & Neutral & Agree & $\begin{array}{c}\text { Strongly } \\
\text { Agree }\end{array}$ \\
\hline $\begin{array}{l}\text { The study results will demonstrate the } \\
\text { benefit of POC time-saving in the ED }\end{array}$ & 1 & 2 & 3 & 4 & 5 \\
\hline
\end{tabular}

7. ANY FURTHER COMMENTS OR THOUGHTS?

\section{THANK YOU FOR YOUR TIME TO COMPLETE THIS SURVEY!}

